\title{
Whole exome sequencing identifies a novel variant in ABCA3 in an individual with fatal congenital surfactant protein deficiency
}

\author{
Hayrunnisa Bekis Bozkurt ${ }^{1 \oplus}$, Yavuz Şahin ${ }^{2 \oplus}$ \\ ${ }^{1}$ Department of Pediatrics, Kafkas University Faculty of Medicine, Kars; ${ }^{2}$ Genox Genetic Diseases Diagnostic Center, Ankara, Turkey.
}

\begin{abstract}
Background. Adenosine triphosphate-binding cassette subfamily A member 3 (ABCA3) gene variants, which cause severe respiratory distress syndrome (RDS) in term newborns, can cause death, especially due to the lack of congenital surfactant protein. The relationship between the types, pathophysiology and effects of ABCA3 gene variants on surfactant metabolism and the clinical phenotype have not yet been fully clarified, but the ABCA3 genotype is known to affect clinical severity.
\end{abstract}

Case. In our study, in a term newborn with a diagnosis of RDS resulting in death, we detected the c.3677 T> C (p.Leu1226Pro) variant homozygous variant in the ABCA3 gene according to the NM_001089.3 transcript, which, to our knowledge, was identified for the first time in the literature.

Conclusions. We consider that this case report contributes to the literature on RDS by showing the presence of c.3677 T> C (p.Leu1226Pro), a new homozygous variant of ABCA3 in our patient.

Key words: ATP-binding cassette transporter, subfamily A; neonatal respiratory distress syndrome, surfactant protein B deficiency, gene variant.

Adenosine triphosphate-binding cassette subfamily A member 3 (ABCA3) is an important glycoprotein expressed in the membrane of type 2 alveolar cells and plays a role in lung surfactant metabolism. ${ }^{1}$ Type 2 alveolar cells are essential for surfactant synthesis and release. Surfactant is a phospholipid and protein complex that is vital in transferring ventilation from the placenta to the postnatal lungs in the intrauterine period and prevents lung collapse by increasing the alveolar surface tension. ${ }^{2}$ To date, more than 200 mutations have been reported for $\mathrm{ABCA}$, which plays a critical role in lipid transport for surfactant synthesis and release. ${ }^{3}$ Biallelic variants in ABCA3 are known to cause autosomal recessive (AR) Pulmonary Surfactant Metabolism Dysfunction type 3 (OMIM \#610921). ${ }^{3}$

\footnotetext{
$凶$ Hayrunnisa Bekis Bozkurt

hayrunisabekis@hotmail.com
}

Received 4th May 2020, revised 12th August 2020,

29th November 2020, accepted 14th January 2021.
Understanding the genetic pathophysiology of the ABCA 3 molecule will open new doors in diagnosis, treatment and management. In our study, we detected the c.3677 T> C (p.Leu1226Pro) variant homozygous variant in the ABCA3 gene according to the NM_001089.3 transcript, which, to our knowledge, has not previously been available in the literature. We aimed to discuss the conditions of our patient during the follow-up and treatment process and the gene variant we detected in order to shed light on this issue.

\section{Case Report}

This paper presents the case of a male infant born at 38 gestational weeks and 2,680 grams by C-section from a 23-year-old healthy mother. It was her second pregnancy and second birth. In his family history, his mother and father were first cousins and he had a 2.5-year-old brother followed up with the diagnosis of 
non-classical congenital adrenal hyperplasia. In addition, the twin children of the patient's aunt were followed up with the diagnosis of primary ciliary dyskinesia, and his aunt also had a consanguineous marriage. The pedigree of the family is shown in Figure 1A. The infant's APGAR score was 7 at the first minute and 8 at the fifth minute. In the delivery room, due to the infant having tachypnea and expiratory grunting, he was admitted to the intensive care unit with the initial diagnosis of transient tachypnea of the newborn (TTN). When the patient was admitted to the intensive care, no pathology was detected in the electrolyte, complete blood count, C-reactive protein (CRP) and blood gas parameters but he had intercostal retractions, expiratory grunting and nasal continuous positive airway pressure (CPAP) treatment was started. Because there was no chorioamnionitis and prolonged premature rupture of membranes and the delivery was a term, the baby was considered to be at low risk for sepsis. Pneumonia was not considered in the foreground because the CRP was negative and there was no infiltration image in favor of pneumonia on chest radiography. Although the first X-ray did not have a significant aeration excess and diaphragmatic flattening; the absence of signs compatible with RDS such as lack of aeration, increased air bronchograms, prominent reticulogranular opacities and the baby's birth was term via C-section this made us think of TTN and we started respiratory support and total parenteral nutrition support first. But, with clinical need; $\mathrm{fiO}_{2}$ was increased as the patient's saturation decreased in the following hours. However, the clinical severity gradually increased and there was no improvement, he was intubated on the postnatal 2nd day. Thereupon, we moved away from the TTN diagnosis and investigate the other potential causes of respiratory distress.
A

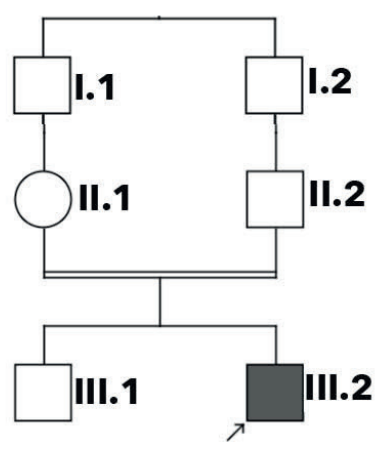

B

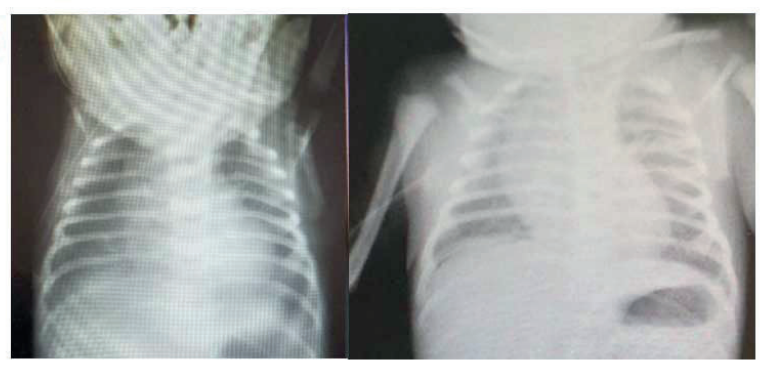

C
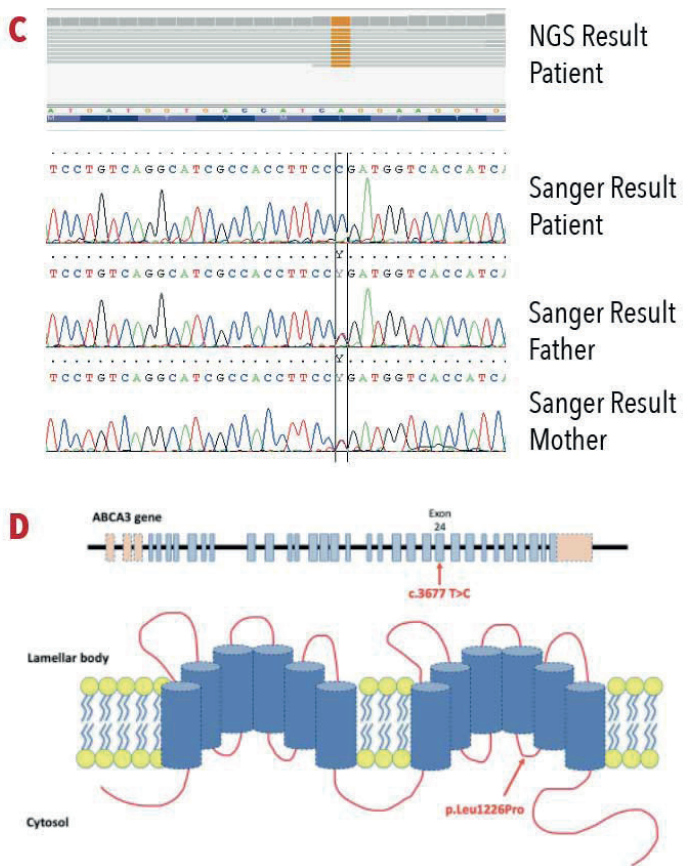

Fig. 1. A) Pedigree of the family, B) X-ray of the chest. First X-ray on the first day of hospitalization and on 3rd day before surfactant treatment, C) Display of Integrative Genomics Viewer showing novel ABCA3 c.3677 $\mathrm{T}>\mathrm{C}$ missense variant in homozygous state in patient while their unaffected parents in heterozygous state. The gray letters and brown letters represent the wild type nucleotides even so the brown letters represent a single base-nucleotide alteration. Parts of the Sanger sequencing electropherograms are depicted and demonstrate homozygosity for the variant c.3677 T>C in patient, heterozygosity in father and mother, D) Structure of the $\mathrm{ABCA} 3$ gene, coded protein, protein domains. Red arrow marks the present variant. 
In terms of persistent pulmonary hypertension, the postnatal 2nd day echocardiography was normal, and there were no findings on the X-ray that were thought to be consistent with pneumothorax or diaphragmatic hernia. For asphyxia, no pathology was found in the blood gas or neurological examination. The absence of clinical improvement on the postnatal 3rd day, the need for intubation, the onset of air bronchograms and the onset of lack of aeration on the X-ray excluded the diagnosis of TTN (Fig. 1B). From this point of view, differential diagnosis of malignant TTN, sepsis, congenital surfactant protein deficiency was considered. Blood values and CRP value $(0.2 \mathrm{mg} / \mathrm{dL})$ were normal, a blood sample was taken from the patient for whole exome sequencing analysis and sent to a genetic laboratory in Ankara, Turkey and $100 \mathrm{mg} / \mathrm{kg}$ surfactant was administered to the patient. Surfactant treatment $(100 \mathrm{mg} /$ $\mathrm{kg}$ ) was administered endotracheally three times on the 3rd, 7th and 12th days. During his follow-up, the patient was fed with total parenteral nutrition. Although there was no growth in the blood culture sent on the first day of the patient's hospitalization, on the 5th day, blood culture, urine culture and control CRP value were sent again as there was no clinical improvement. The patient was started on broad spectrum antibiotic therapy (ampicillin + gentamicin combination) with suspected sepsis pre-diagnosis because CRP was 0.7 $\mathrm{mg} / \mathrm{dL}(>0.5 \mathrm{mg} / \mathrm{dL}$ ). Antibiotic treatment was stopped on the seventh day. Abdominal and transfontanelle ultrasonography was performed routinely on the seventh postnatal day and revealed normal findings. Upon the development of pulmonary hypertension on the 20th day, inhaled nitric oxide and sildenafil combination treatment was started. However, the patient was still dependent on intubation. Despite all the supportive treatments on the 24th day of his life, he died due to severe RDS and respiratory failure. The family was referred to genetic counseling. Written consent was obtained from the patient's family to publish this report.

\section{Genetic analyses}

Genomic DNA was extracted from peripheral blood cells according to the manufacturer's standard procedure (DNeasy Blood \& Tissue Kits - QIAGEN). gDNA was broken into 150-500 bp fragments using a BGI enzymes kit (Segmentase, BGI), The fragments were collected using magnetic beads. Extracted DNA was amplified using a ligation-mediated polymerase chain reaction (LM- PCR). A mean exome coverage of more than $99 \%$ was obtained. The sequencing depth was greater than $100 \times$ for capture regions. Lastly, the qualified products were sequenced with PE100 +100 on MGISEQ-2000 (BGI, China).

The raw data obtained was aligned with the Burrows-Wheeler Aligner (BWA) algorithm. SamTools and Picard programs were used to sort and discard polymerase chain reaction duplications. Genome analysis toolkit (GATK) program v. 3.7 was used to invoke variants. ANNOVAR, (a bioinformative tool algorithm, was used to reveal the variants, and the raw data was prepared for analysis.

Variants were filtered based on frequency, inheritance pattern, clinical phenotype and pathogenicity. After that, the c.3677 T> C (p.Leu1226Pro) variant was detected to be homozygous according to the NM_001089.3 transcript in the ABCA3 gene, which was considered to explain the patient's clinical state. Sanger sequencing was performed to verify the variant at proband and parents. There was no significant variant in primary ciliary dyskinesia genes recorded at OMIM database.

\section{Discussion}

In our study, we detected the c.3677 T> C (p.Leu1226Pro) variant as homozygous in the ABCA3 gene according to the NM_001089.3 transcript. To our knowledge, this variant is novel. The child had inherited the variant from the unaffected mother and father, both in a heterozygous state (Fig. 1C,1D). The identified genetic variation was not found in any databases, 
including the1000Genome projects, esp6500, ExAC, gnom AD, and our in-house database ( $\mathrm{n}=1978$ Turkish individuals). At the same time, it is located in a highly conserved residue and GERP score showed the mutated region is conserved among the species. Bioinformatic Prediction analysis with in silico algorithms, such as Mutation Taster, Polyphen-2, CADD, Revel, M-CAP, and SIFT showed this alteration to be pathogenic and disease causing. ${ }^{4-7}$

Missense ABCA3 gene variants are thought to cause disruption in intracellular $\mathrm{ABCA} 3$ protein traffic and therefore cause disease. ${ }^{8}$ This missense variant is located in the intracellular loop, which deviates in intracellular interactions that probably have a severe effect on protein function. We demonstrated the theoretical 3D structure of protein and mutant amino acid residue by using HOPE. HOPE analysis revealed the alteration can affect the protein function. (https://www3.cmbi.umcn.nl/hope/ progress/5e9f34796f82a694596d3fa1/).

Mitsiakos et al. ${ }^{9}$ reported a case with a missense variant located in exon 23 of the ABCA3 gene affecting intracellular interactions with total (surfactant) deficiency and fatal early onset. Our patients missense variant located in exon 24 resulted in the substitution of a neutral leucine residue for a non-polar hydrophobic proline residue at position 1226 (p.Leu1226Pro) which can affect intracellular interactions. The variant was submitted to the CLINVAR database (Submission ID: SUB7881781).

In a study by Wambach et al. ${ }^{10}$, it was found that the p.R288K and p.R1474W variants caused severe neonatal RDS by reducing the ATPase activity while the c.875A $>\mathrm{T}$ (p.Glu292Val) variant was associated with a milder form of the disease. Another study showed that missense variants in the ABCA3 gene could be molecular methods, and the p.Q215K and p.R288K variants led to early death and severe RDS. ${ }^{1,11}$ The survival of biallelic ABCA3 variants until adulthood has rarely been observed, and they are shown to present as interstitial lung injury. However, the variant of these patients are of partial, small frame types that do not lead to the loss of total function. ${ }^{12}$ In a study in which 185 infants and children with ABCA3 variants were included; frameshift and nonsense variants were classified as 'null', while splice site, insertion, deletion, missense variants were classified as 'other' and all individuals with 'null/null' variants died under the age of 1 . However, in this study, it was observed that only $36 \%$ of those with a 'null / other' or 'other/ other' variation, survived after the age of $1 .{ }^{13} \mathrm{We}$ detected the missense mutation as homozygous in our patient and our patient died in the neonatal period.

In the differential diagnosis of term newborn RDS, primary pulmonary hypertension, diabetic mother, primary ciliary dyskinesia, congenital surfactant protein deficiencies or hereditary disorders causing congenital surfactant deficiency should be considered. ${ }^{14}$ $A B C A 3$ gene variants lead to the deficiency of surfactant proteins B and C. A recent study showed that hemagglutinin-tagged wild type ABCA3 and its variants p.Q215K, p.A1046E, p.K1388N, p.G1421R can be corrected, and all mutant proteins except M760R ABCA3 are rescued in vitro. ${ }^{15}$ Although genetic therapies are promising for the future, lethal RDS due to congenital surfactant metabolism disorder still has no effective treatment other than lung transplantation. ${ }^{14}$ The DNA sequence analysis of our patient was completed approximately 15 days after his death. The patient was connected to mechanical ventilation and given supportive therapy.

In conclusion, we consider that this case report contributes to the literature on RDS by showing the presence of c.3677 T> C (p.Leu1226Pro), a novel homozygous variant of ABCA3 in our patient. Further studies are needed to explain the in vitro genetic pathways of $\mathrm{ABCA} 3$ variants. Such studies are important for future therapeutic studies and the determination of treatment alternatives to lung transplantation. 


\section{Author contribution}

The authors confirm contribution to the paper as follows: study conception and design: HBB, YŞ; data collection: HBB; analysis and interpretation of results: $\mathrm{HBB}, \mathrm{YSS}$; draft manuscript preparation: HBB, YŞ. All authors reviewed the results and approved the final version of the manuscript.

\section{Conflict of interest}

None.

\section{REFERENCES}

1. Kröner C, Wittmann T, Reu S, et al. Lung disease caused by ABCA3 mutations. Thorax 2017; 72: 213220.

2. Rindler TN, Stockman CA, Filuta AL, et al. Alveolar injury and regeneration following deletion of ABCA3. JCI Insight 2017; 2: e97381.

3. Beers MF,Mulugeta S. The biology of the ABCA3 lipid transporter in lung health and disease. Cell Tissue Res 2017; 367: 481-493.

4. Adzhubei IA, Schmidt S, Peshkin L, et al. A method and server for predicting damaging missense mutations. Nat Methods 2010; 7: 248-249.

5. Kircher M, Witten DM, Jain P, O'Roak BJ, Cooper GM, Shendure J. A general framework for estimating the relative pathogenicity of human genetic variants. Nat Genet 2014; 46: 310-315.

6. Jagadeesh KA, Wenger AM, Berger MJ, et al. M-CAP eliminates a majority of variants of uncertain significance in clinical exomes at high sensitivity. Nat Genet 2016; 48: 1581-1586.
7. Schwarz JM, Cooper DN, Schuelke M, Seelow D. MutationTaster2: mutation prediction for the deepsequencing age. Nat Methods 2014; 11: 361-362.

8. Cheong N, Madesh M, Gonzales LW, et al. Functional and trafficking defects in ATP binding cassette A3 mutants associated with respiratory distress syndrome. J Biol Chem 2006; 281: 9791-9800.

9. Mitsiakos G, Tsakalidis C, Karagianni P, et al. A new ABCA3 gene mutation c. 3445G> a (p. Asp1149Asn) as a causative agent of newborn lethal respiratory distress syndrome. Medicina (Kaunas) 2019; 55: 389.

10. Wambach JA, Yang P, Wegner DJ, et al. Functional characterization of ATP-binding cassette transporter A3 mutations from infants with respiratory distress syndrome. Am J Respir Cell Mol Biol 2016; 55:716721.

11. Schindlbeck U, Wittmann T, Höppner S, et al. ABCA3 missense mutations causing surfactant dysfunction disorders have distinct cellular phenotypes. Hum Mutat 2018; 39: 841-850.

12. Bullard JE, Wert SE, Whitsett JA, Dean M, Nogee LM. ABCA3 mutations associated with pediatric interstitial lung disease. Am J Respir Crit Care Med 2005; 172: 1026-1031.

13. Wambach JA, Casey AM, Fishman MP, et al Genotype-phenotype correlations for infants and children with ABCA3 deficiency. Am J Respir Crit Care Med2014; 189: 1538-1543.

14. Chowdhury N, Giles BL, Dell SD. Full-term neonatal respiratory distress and chronic lung disease. Pediatr Ann 2019; 48: e175-e181.

15. Kinting S, Höppner S, Schindlbeck U, et al. Functional rescue of misfolding ABCA3 mutations by small molecular correctors. Hum Mol Genet 2018; 27: 943-953. 